\title{
THE CANONICAL CONTROLLER and ITS REGULARITY
}

\author{
Jan C. Willems \\ University of Leuven \\ Kasteelpark Arenberg 10 \\ B-3001 Leuven-Heverlee Belgium \\ Jan. Willemsesat. kuleuven. ac.be \\ www. esat. kuleuven.ac.be/ jwillems
}

\section{A. Agung Julius \\ University of Twente \\ P. O. Box 217}

7500 AE Enschede The Netherlands

A.A.Julius@math.utwente.nl

www. math.utwente.nl/ juliusaa

\author{
Madhu N. Belur \\ University of Groningen \\ P.O. Box 800 \\ 9700 AV Groningen The Netherlands \\ M.N.Beluramath.rug.nl \\ www. math. rug.nl/ madhu \\ Harry L. Trentelman
University of Groningen \\ P.O. Box 800 \\ 9700 AV Groningen The Netherlands \\ H.I. Trentelmandmath.rug.nl \\ www. math.rug.nl/ trentelman
}

\begin{abstract}
This paper deals with properties of canonical controllers. We first specify the behavior that they implement. It follows that a canonical controller implements the desired controlled behavior if and only if the desired behavior is implementable. We subsequently investigate the regularity of the controlled behavior. We prove that a canonical controller is regular if and only if every controller is regular. In other words, canonical controllers are maximally irregular.
\end{abstract}

Keywords: Behaviors, behavioral control, regular interconnection, regular controller, canonical controller, implementability.

\section{CONTROL IN A BEHAVIORAL SETTING}

It is common in control theory to view a controller as a feedback processor that accepts the plant sensor outputs as its inputs and produces the actuator inputs as its outputs. We like to call 'intelligent control': the controller acts as an artificially intelligent device that reasons how to react to sensory observations. In behavioral control, on the other hand, the idea is to view a controller as a subsystem that is designed with the purpose of achieving good performance of the overall system in which it is embedded.

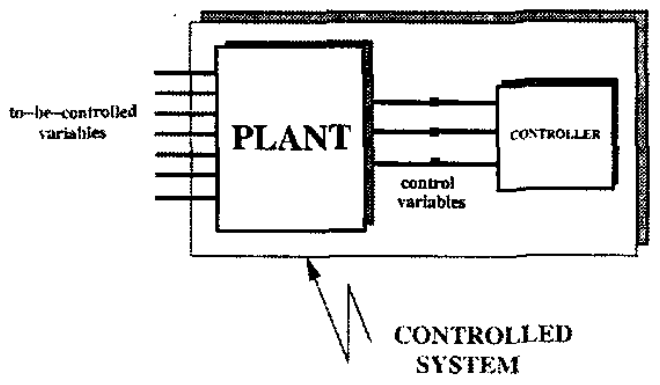

Fig. 1. Control as interconnection

More concretely, we start with a (to-be-controlled) plant, having two kinds of variables: to-be-controlled variables and control variables. A controller is a device that acts on the control variables, and restricts their behavior. This restriction is transmitted through the plant to the to-be-controlled variables. The resulting system (i.e. the behavior of the to-becontrolled variables with the controller attached) is called the controlled system. It is the behavior of this system that should meet the control specifications. This control architecture is shown in figure 1.

The main advantages of the behavioral over the classical feedback point of view, are (i) its practical generality: many control devices do not act as sensor/actuator devices (dampers, heat fins, acoustic noise insulators, appendages to enhance aerodynamic properties, etc., etc.), and (ii) its theoretical simplicity. Control in a behavioral setting has been introduced in [10] and further developed in [4], [8], [12]. We refer to these references and to [13] for further motivation and details.

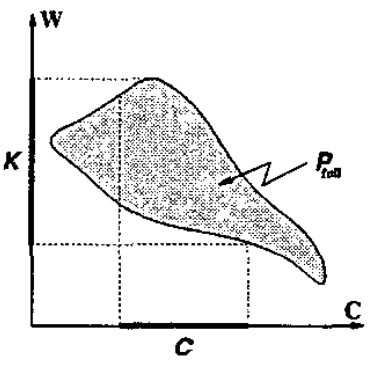

Fig. 2. The controlled behavior

The formal definitions of the plant, controller, and controlled behavior are as follows. Let $\mathbf{W}$ and $\mathbf{C}$ denote the set of all signals $w$ and $c$ that are a priori possible, before we even modelled the plant. In dynamical systems, $\mathbf{W}$ and $\mathbf{C}$ are typically the set of (smooth) signals from the time axis to the signal spaces $\mathbb{W}$ of the to-be-controlled variables, and $\mathbb{C}$ of the control variables. In DES, $W$ and $C$ are typically all words with letters from alphabets $\mathbb{W}$ and $\mathbb{C}$. 
The full plant behavior is a subset $\mathcal{P}_{\text {full }}$ of $\mathbf{W} \times \mathbf{C}$ : it consists of those signals $(w, c)$ compatible with the plant dynamics. A controller $\mathcal{C}$ is a subset of $\mathbf{C}$ : it consists of those signals $c$ which the controller allows. The controlled behavior is then defined by

$$
\begin{aligned}
\mathcal{K}:=\{w \in \mathbf{W} \mid & \exists c \in \mathbf{C} \\
& \text { such that } \left.(w, c) \in \mathcal{P}_{\text {full }} \text { and } c \in \mathcal{C}\right\} .
\end{aligned}
$$

This definition of $\mathcal{K}$ is illustrated in figure 2 . If, for a given full plant behavior $\mathcal{P}_{\text {full, }}$, there exists a controller $\mathcal{C}$ such that the resulting controlled system equals $\mathcal{K}$, then we call $\mathcal{K}$ implementable, or implemented by $\mathcal{C}$.

The controller synthesis problem is to find, for a given plant with behavior $\mathcal{P}_{\text {full, }}$ a controller $\mathcal{C}$ such that the resulting controlled behavior $\mathcal{K}$ meets certain performance specifications. In this paper, we will take this to mean that there is a desired controlled behavior $\mathcal{D} \subseteq \mathrm{W}$ and that the control synthesis requirement is $\mathcal{K}=\mathcal{D}$.

\section{THE CANONICAL CONTROLLER}

The basic goal of the controller is to achieve a certain desired behavior of the to-be-controlled variables. The problem thus arises:

\section{Given a plant and a desired behavior, choose a controller that achieves this.}

In a recent paper [5], [6], van der Schaft proposed an eminent, universal candidate controller. It is constructed by taking the plant and attaching (on the side of the to-becontrolled variables!) the desired controlled system to it, as shown in figure 3 . Note that since in the canonical controller,

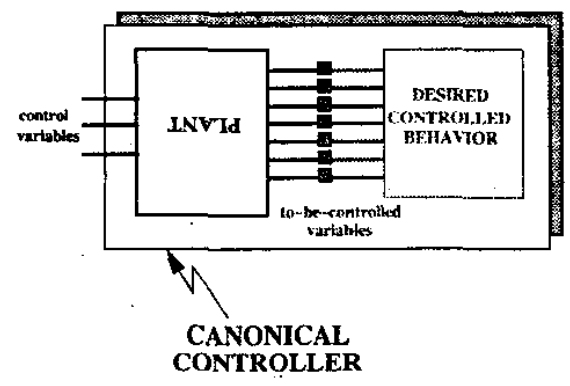

Fig. 3. The canonical controller

the terminals of the plant are reversed, we marked PLANT upside-down (the mirror image, unavailable, would have been better). Connecting this controller to the plant leads to the controlled system shown in figure 4 .

The definition of this canonical controller $\mathcal{C}_{\text {canonical is }}^{\prime}$

$$
\begin{aligned}
\mathcal{C}_{\text {canonical }}^{\prime}:=\{c \in \mathbf{C} \mid \exists v & \in \mathcal{D} \text { such that } \\
& \left.(v, c) \in \mathcal{P}_{\text {full }} \text { and } v \in \mathcal{D}\right\} .
\end{aligned}
$$

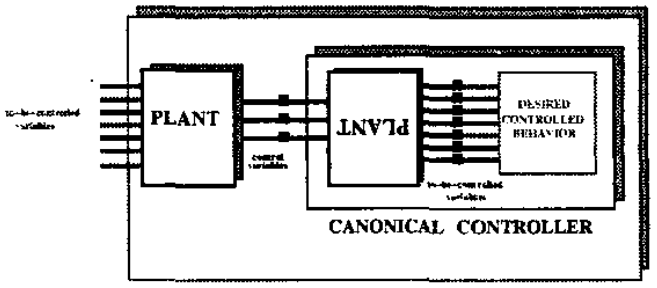

Fig. 4. The canonically controlled systems

However, there is a second canonical controller, $\mathcal{C}^{\prime \prime}$ canonical, that has better properties. It is defined by

$$
\begin{aligned}
& \mathcal{C}_{\text {canonical }}^{\prime \prime}:=\left\{c \in \mathrm{C} \mid \exists v \text { such that }(v, c) \in \mathcal{P}_{\text {full }},\right. \\
& \text { and } \left.(v, c) \in \mathcal{P}_{\text {full }} \Rightarrow v \in \mathcal{D}\right\} .
\end{aligned}
$$

The action of the second canonical controller is shown in figure 5 , where we have replaced the connectors by symbols suggesting 'implies'.

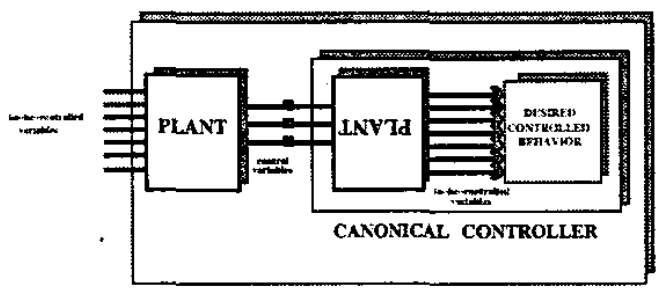

Fig. 5. The second canonical controller

The canonical controllers have all the features of a controller that is based on an internal model. Indeed, in deciding how to constrain the control variables, the canonical controllers achieve this by transmitting the imposed specification on the to-be-controlled variables through the plant to the control variables. The canonical controllers are a marvellous idea. The action of these canonical controllers is illustrated in figure 6. It is easy to see that these canonical controllers both

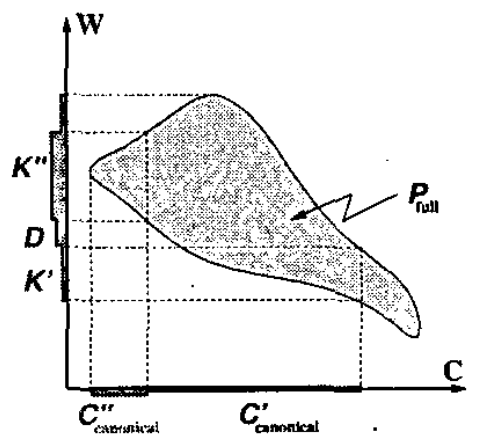

Fig. 6. The action of the canonical controllers

implement $\mathcal{D}$ if and only if it is implementable. Moreover, the controlled behavior implemented by the second canonical 
controller is actually the largest implementable controlled behavior contained in $\mathcal{D}$.

In [5], [6] a number of the properties of the first canonical controller have already been discussed. In the present article, we go more deeply into these properties for linear timeinvariant systems.

\section{IMPLEMENTABILITY}

We will henceforth restrict attention to linear timeinvariant differential systems. We refer to [9], [4], [13] for an extensive introduction to this class of systems. We will freely use the following notation that has become standard in this area. $\mathfrak{L}^{\mathrm{y}}$ denotes the class of linear time-invariant differential systems with $w$ variables. Thus by definition of

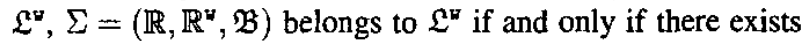
a polynomial matrix $R \in \mathbb{R}^{\bullet \times v}[\xi]$ such that the behavior $\mathfrak{B}$ is the solution set of the system of differential equations

$$
R\left(\frac{d}{d t}\right) w=0
$$

Concretely, $\mathfrak{B}$ is defined by

$$
\mathfrak{B}=\left\{w \in \mathfrak{C}^{\infty}\left(\mathbb{R}, \mathbb{R}^{w}\right) \mid R\left(\frac{d}{d t}\right) w=0\right\} .
$$

We often write this as $\mathfrak{B} \in \mathfrak{L}^{\mathfrak{w}}$ instead of $\Sigma \in \mathfrak{L}^{w}$. Often, a behavior is defined in terms of auxiliary variables. In this case, we use the term manifest for the variables of interest, and latent for the auxiliary variables. If $\mathfrak{B} \in \mathfrak{L}^{\boldsymbol{y}+\ell}$ is a system involving the manifest variables $w \in \mathfrak{C}^{\infty}\left(\mathbb{R}, \mathbb{R}^{w}\right)$ and the latent variables $\ell \in \mathfrak{C}^{\infty}\left(\mathbb{R}, \mathbb{R}^{\ell}\right)$, then it turns out that the manifest behavior $\mathfrak{B}_{\mathfrak{v}}$ defined by

$$
\mathfrak{B}_{\mathfrak{w}}:=\left\{w \in \mathfrak{C}^{\infty}\left(\mathbb{R}, \mathbb{R}^{*}\right) \mid \exists \ell \in \mathfrak{C}^{\infty}\left(\mathbb{R}, \mathbb{R}^{\ell}\right):(w, \ell) \in \mathfrak{B}\right\}
$$

is an element of $\mathfrak{L}^{w}$. This result, that the projection of a differential behavior is also a differential behavior, is called the elimination theorem, and of one of the central results in the theory of differential systems (see [9], or [4, section 6.2]). The $\mathfrak{C}^{\infty}$-assumption is made mainly for convenience, and the results do not depend on this assumption (see [4, chapter 2] for a discussion of this issue). The differential equation $R\left(\frac{d}{d t}\right) w=0$ is called a kernel representation for $\mathfrak{B}$. Sometimes, we use the notation $\operatorname{ker}\left(R\left(\frac{d}{d t}\right)\right)$ for $\mathfrak{B}$. A kernel representation is called minimal if and only if $R$ has full row rank (meaning that its rank is equal to its number of rows).

We now turn to the control problem. Consider the plant

$$
\Sigma_{\text {plant }}=\left(\mathbb{R}, \mathbb{R}^{w} \times \mathbb{R}^{c}, \mathcal{P}_{\text {full }}\right) \in \mathfrak{L}^{k+c} .
$$

Hence the plant behavior $\mathcal{P}_{\text {full }}$ constrains the to-be-controlled variables $w$ and the control variables $c$ by a system of linear constant coefficient differential equations. The controller is now assumed to be a system

$$
\Sigma_{\text {controller }}=\left(\mathbb{R}, \mathbb{R}^{\mathrm{c}}, \mathcal{C}\right) \in \mathfrak{L}^{\mathrm{c}} .
$$

Hence the controller behavior $\mathcal{C}$ constrains the control variables $c$ by a system of linear constant coefficient differential equations. The controlled system is

$$
\Sigma_{\text {controlled }}=\left(\mathbb{R}, \mathbb{R}^{*}, \mathcal{K}\right),
$$

with the controlled behavior $\mathcal{K}$ defined by

$\mathcal{K}=\left\{w \in \mathfrak{C}^{\infty}\left(\mathbb{R}, \mathbb{R}^{*}\right) \mid \exists c \in \mathcal{C}\right.$ such that $\left.(w, c) \in \mathcal{P}_{\text {full }}\right\}$.

As a consequence of the elimination theorem, $\Sigma_{\text {controlled }} \in \mathfrak{L}^{\natural}$. Hence $\mathcal{K}$ is also governed by a system of linear constant coefficient differential equations. If, for a given $\Sigma_{\text {plant }}, \Sigma_{\text {controller }}$ leads to $\Sigma_{\text {controlled, then we say the }}$ $\Sigma_{\text {controlled }}$ is implemented by $\Sigma_{\text {controller }}$, and that $\Sigma_{\text {controlled }}$ is implementable. The question arises

Which behaviors $\mathcal{K} \in \mathfrak{L}^{\text {u }}$ can be implemented by attaching a suitable controller $\mathcal{C} \in \mathfrak{L}^{\mathrm{c}}$ to a given $\mathcal{P}_{\text {full }} \in \mathfrak{L}^{\mathrm{r}+\mathrm{c}}$ ?

This question has a very concrete and intuitive answer.

Theorem 1: Let $\mathcal{P}_{\text {full }} \in \mathfrak{L}^{w+c}$ be given. The behavior $\mathcal{K} \in \mathfrak{L}^{w}$ is implementable if and only if

$$
\mathcal{N} \subseteq \mathcal{K} \subseteq \mathcal{P}
$$

where $\mathcal{N} \in \mathfrak{L}^{w}$ is the hidden behavior defined by

$$
\mathcal{N}:=\left\{w \in \mathfrak{C}^{\infty}\left(\mathbb{R}, \mathbb{R}^{w}\right) \mid(w, 0) \in \mathcal{P}_{\text {full }}\right\},
$$

and $\mathcal{P}$ is the manifest plant behavior defined by

$$
\mathcal{P}:=\left\{w \in \mathfrak{C}^{\infty}\left(\mathbb{R}, \mathbb{R}^{w}\right) \mid \exists \text { c such that }(w, c) \in \mathcal{P}_{\text {full }}\right\} .
$$

Note that it follows from the elimination theorem that $\mathcal{N}, \mathcal{P} \in \mathfrak{L}^{*}$. This theorem reduces (linear) control questions to finding a subspace that is wedged in between two given subspaces. This simple characterization was obtained after [10], first announced in [11], has since been pursued in a number of publications [3], [7], but the most extensive exposition is given in [12].

We repeat the idea of the proof of the 'if' part (the other direction is trivial), since it is of some relevance to the canonical controller. Let

$$
R\left(\frac{d}{d t}\right) w=M\left(\frac{d}{d t}\right) c
$$

be a kernel representation for $\mathcal{P}_{\text {full }}$. Then $R\left(\frac{d}{d t}\right) w=0$ is obviously a kernel representation of $\mathcal{N}$. Since $\mathcal{N} \subseteq \mathcal{K}$, it follows that $\mathcal{K}$ has a kernel representation of the form $F\left(\frac{d}{d t}\right) R\left(\frac{d}{d t}\right) w=0$ for a suitable $F \in \mathbb{R}^{\bullet \bullet \bullet}[\xi]$. It turns out that

$$
F\left(\frac{d}{d t}\right) M\left(\frac{d}{d t}\right) c=0
$$

is actually a controller that implements $\mathcal{K}$ (the proof of this uses $\mathcal{K} \subseteq \mathcal{P}$ ).

It is important to observe that the controller that implements $\mathcal{K}$ may not be unique, for example, because $F$ may not be unique. So, controllers that implement the same controlled behavior may have very different properties. 


\section{THE CONTROLLED BEHAVIOR IMPLEMENTED BY THE CANONICAL CONTROLLER}

Consider, for a given plant $\mathcal{P}_{\text {full }} \in \mathfrak{L}^{w+c}$, and for a given desired controlled behavior, $\mathcal{D} \in \mathfrak{L}^{\mathrm{w}}$ the associated canonical controllers. The first canonical controller is defined by

$$
\Sigma_{\text {canonical }}^{\prime}:=\left(\mathbb{R}, \mathbb{R}^{c}, \mathcal{C}_{\text {canonical }}^{\prime}\right)
$$

with $\mathcal{C}_{\text {canonical }}^{\prime}$ given by

$$
\begin{aligned}
\mathcal{C}_{\text {canonical }}^{\prime}:=\left\{c \in \mathfrak{C}^{\infty}\left(\mathbb{R}, \mathbb{R}^{c}\right)\right. & \mid \exists v \in \mathcal{D} \\
& \text { such that } \left.(v, c) \in \mathcal{P}_{\text {full }}\right\} .
\end{aligned}
$$

In terms of kernel representations, $\mathcal{C}_{\text {canonical }}^{\prime}$ is the manifest behavior (with $c$ viewed as the manifest variable!) of

$$
R\left(\frac{d}{d t}\right) v=M\left(\frac{d}{d t}\right) c, \quad D\left(\frac{d}{d t}\right) v=0,
$$

with $D\left(\frac{d}{d t}\right) v=0$ a kernel representation of $\mathcal{D}$.

The second canonical controller is defined by

$$
\Sigma_{\text {canonical }}^{\prime \prime}:=\left(\mathbb{R}, \mathbb{R}^{c}, \mathcal{C}_{\text {canonical }}^{\prime \prime}\right)
$$

with $\mathcal{C}_{\text {canonical }}^{\prime \prime} \in \mathfrak{L}^{c}$ given by

$$
\begin{gathered}
\mathcal{C}_{\text {canonical }}^{\prime \prime}:=\left\{c \in \mathfrak{C}^{\infty}\left(\mathbb{R}, \mathbb{R}^{c}\right) \mid \exists v \text { such that }(v, c) \in \mathcal{P}_{\text {full }},\right. \\
\text { and } \left.(v, c) \in \mathcal{P}_{\text {full }} \Rightarrow v \in \mathcal{D}\right\} .
\end{gathered}
$$

For linear time-invariant differential systems there is little difference between these two canonical controllers. In fact,

Lemma: $\mathcal{C}_{\text {canonical }}^{\prime} \in \mathfrak{L}^{c} . \mathcal{C}_{\text {canonical }}^{\prime \prime}$ is non-empty if and only if $\mathcal{N} \subseteq \mathcal{D}$. If $\mathcal{N} \subseteq \mathcal{D}$, then $\mathcal{C}_{\text {canonical }}^{\prime}=\mathcal{C}_{\text {canonical }}^{\prime \prime}$

Proof: We first prove that $c_{1}, c_{2} \in \mathcal{C}_{\text {canonical }}^{\prime \prime} \Rightarrow c_{1}+$ $c_{2} \in \mathcal{C}_{\text {canonical }}^{\prime \prime}$ Assume $\left(c_{1}, c_{2}\right) \in \mathcal{C}_{\text {canonical. Then there is }}^{\prime \prime}$ $w_{1} \in \mathcal{D}$ such that $\left(w_{1}, c_{1}\right) \in \mathcal{P}_{\text {full }}$. Therefore

$\left(w, c_{1}+c_{2}\right) \in \mathcal{P}_{\text {full }}$ $\Rightarrow\left(w-w_{1}, c_{2}\right) \in \mathcal{P}_{\text {full }}$ (since $\mathcal{P}_{\text {full }}$ is linear)

$\Rightarrow\left(w-w_{1}\right) \in \mathcal{D}$ (since $\left.c_{2} \in \mathcal{C}_{\text {canonical }}^{\prime \prime}\right)$

$\Rightarrow w \in \mathcal{D}$ (since $\mathcal{D}$ is linear).

Hence $\left(c_{1}, c_{2}\right) \in \mathcal{C}_{\text {canonical }}^{\prime \prime} \Rightarrow\left(c_{1}+c_{2}\right) \in \mathcal{C}_{\text {canonical }}^{\prime \prime}$.

Next, observe that $c \in \mathcal{C}_{\text {canonical }}^{\prime \prime} \Rightarrow-c \in \mathcal{C}_{\text {canonical }}^{\prime \prime}$ This follows from

$(-w,-c) \in \mathcal{P}_{\text {full }} \Leftrightarrow(w, c) \in \mathcal{P}_{\text {full }} \Rightarrow w \in \mathcal{D} \Leftrightarrow-w \in \mathcal{D}$.

This immediately implies that if $\mathcal{C}_{\text {canonical }}^{\prime \prime}$ is non-empty, then $0 \in \mathcal{C}_{\text {canonical }}^{\prime \prime}$, and $\mathcal{N} \subseteq \mathcal{D}$. The latter is a consequence of $w \in \mathcal{N} \Leftrightarrow(w, 0) \in \mathcal{P}_{\text {full }} \Rightarrow w \in \mathcal{D}$. Hence $\mathcal{C}_{\text {canonical }}^{\prime \prime}$ is non-empty if and only $\mathcal{N} \subseteq \mathcal{D}$.

We now clinch the proof by showing that if $\mathcal{N} \subseteq \mathcal{D}$, then $\mathcal{C}_{\text {canonical }}^{\prime}=\mathcal{C}_{\text {canonical }}^{\prime \prime}$ To see this, assume $c \in \mathcal{C}_{\text {canonical }}^{\prime}$ Then $\exists w \in \mathcal{D}$ such that $(w, c) \in \mathcal{P}_{\text {full }}$. Assume now $\left(w^{\prime}, c\right) \in \mathcal{P}_{\text {full }}$. Then

$\left(w^{\prime}-w, 0\right) \in \mathcal{P}_{\text {full }}$ (since $\mathcal{P}_{\text {full }}$ is linear)

$\Rightarrow\left(w^{\prime}-w\right) \in \mathcal{N}$ (by the definition of $\mathcal{N}$ )

$\Rightarrow\left(w^{\prime}-w\right) \in \mathcal{D}$ (since $\mathcal{N} \subseteq \mathcal{D}$ )

$\Rightarrow w^{\prime} \in \mathcal{D}$ (since $\mathcal{D}$ is linear).
Hence $c \in \mathcal{C}_{\text {canonical }}^{\prime \prime}$, and $\mathcal{C}_{\text {canonical }}^{\prime} \subseteq \mathcal{C}_{\text {canonical }}^{\prime \prime}$ The converse $\mathcal{C}_{\text {canonical }}^{\prime \prime} \subseteq \mathcal{C}_{\text {canonical }}^{\prime}$ is obvious.

This ends the proof of the lemma.

Motivated by this lemma, we need henceforth only consider the first canonical controller $\mathcal{C}_{\text {canonical }}^{\prime}$. Note that the canonical controller is well-defined even when $\mathcal{D}$ is not implementable. The question what controlled behavior is actually implemented by the canonical controller is settled in the following theorem.

Theorem 2: Consider $\mathcal{P}_{\text {full }} \in \mathfrak{L}^{\mathbf{w}+c}$ and $\mathcal{D} \in \mathfrak{L}^{\mathrm{w}}$. The controlled behavior implemented by the associated canonical controller $\mathcal{C}_{\text {canonical }}^{\prime} \in \mathfrak{L}^{\mathfrak{c}}$ is

$$
\mathcal{K}=\mathcal{N}+\mathcal{D} \cap \mathcal{P}
$$

with $\mathcal{N}$ the hidden and $\mathcal{P}$ the manifest plant behavior.

Proof: The implemented controlled behavior is the manifest $w$-behavior of

$$
\begin{array}{ll}
\mathcal{P}_{\text {full }}: & R\left(\frac{d}{d t}\right) w=M\left(\frac{d}{d t}\right) c \\
\mathcal{C}_{\text {canonical }}^{\prime}: & R\left(\frac{d}{d t}\right) v=M\left(\frac{d}{d t}\right) c, \quad D\left(\frac{d}{d t}\right) v=0
\end{array}
$$

This has the same manifest $w$ behavior as

$$
\begin{aligned}
& R\left(\frac{d}{d t}\right) v=M\left(\frac{d}{d t}\right) c, \\
& R\left(\frac{d}{d t}\right)(w-v)=0, \quad D\left(\frac{d}{d t}\right) v=0
\end{aligned}
$$

Now define $w^{\prime}=w-v$, and obtain

$$
\begin{array}{ll}
\mathcal{N}: & R\left(\frac{d}{d t}\right) w^{\prime}=0 \\
\mathcal{D} \cap \mathcal{P}: & D\left(\frac{d}{d t}\right) v=0, \quad R\left(\frac{d}{d t}\right) v=M\left(\frac{d}{d t}\right) c \\
\mathcal{N}+\mathcal{D} \cap \mathcal{P} & w=w^{\prime}+v .
\end{array}
$$

This shows that indeed $\mathcal{K}=\mathcal{N}+\mathcal{D} \cap \mathcal{P}$.

The above theorem Ieads to the following corollary. It shows that the canonical controller always implements a desired controlled behavior, provided it is implementable.

Corollary: The canonical controller implements $\mathcal{D} \in \mathfrak{L}^{\mathrm{u}}$ if and only if $\mathcal{D}$ is implementable, i.e. if and only if $\mathcal{N} \subseteq$ $\mathcal{D} \subseteq \mathcal{P}$.

\section{REGULAR CONTROLLERS}

Consider $\mathfrak{B} \in \mathfrak{L}^{\Downarrow}$. Then it is well-known (see [9], [4]) that the variables $\left(w_{1}, w_{2}, \ldots, w_{\mathfrak{v}}\right)$ in $\mathfrak{B}$ allow a component-wise partition into free inputs and bound outputs. This input/output partition is put into evidence by the kernel representation

$$
P\left(\frac{d}{d t}\right) y=Q\left(\frac{d}{d t}\right) u, \quad w=(u, y)
$$

with $P, Q \in \mathbb{R}^{\bullet \bullet \bullet}[\xi], P$ square and $\operatorname{det}(P) \neq 0$. In fact, the partition can even be chosen so that the transfer function $G=P^{-1} Q$ is proper. In this input/output partition of the $w$ variables, $u$ and $y$ are not unique, but the number of input and output variables is invariant, i.e., this number is independent of the input/output partition, while the variables themselves are not.

This leads to two maps $\mathrm{m}, \mathrm{p}: \mathfrak{L}^{\mathfrak{w}} \rightarrow\{0,1, \ldots, w\}$ with $m(\mathfrak{B})$ the number of inputs, and $p(\mathfrak{B})$ the number of output 
variables in $\mathfrak{B}$, and $\mathrm{m}+\mathrm{p}=\boldsymbol{w}$. In terms of a kernel representation $R\left(\frac{d}{d t}\right) w=0$ of $\mathfrak{B}, \mathrm{p}(\mathfrak{B})=\mathrm{w}-\mathrm{m}(\mathfrak{B})=$ $\operatorname{rank}(R)$.

Recall that for a given plant $\mathcal{P}_{\text {full }} \in \mathfrak{L}^{\mathrm{w}+\mathrm{c}}$ and a given controller $\mathcal{C} \in \mathfrak{L}^{c}$ we defined the manifest controlled behavior $\mathcal{K}$. In this section, we also need the full controlled behavior $\mathcal{K}_{\text {full }} \subseteq \mathcal{P}_{\text {full }}$ defined by

$$
\mathcal{K}_{\text {full }}:=\left\{(w, c) \in \mathcal{P}_{\text {full }} \mid c \in \mathcal{C}\right\} .
$$

The controller $\mathcal{C} \in \mathfrak{L}^{\mathrm{c}}$ is said to be regular if the following relation holds

$$
\mathrm{p}\left(\mathcal{K}_{\text {full }}\right)=\mathrm{p}\left(\mathcal{P}_{\text {full }}\right)+\mathrm{p}(\mathcal{C})
$$

Note that in a sense this means that the plant and the controller equations combined are independent of each other. It can be shown that a controller is regular if and only if it can actually be realized as a (possibly non-proper) transfer function from an output variable to an input variable in $\mathcal{P}_{\text {full }}$ for an input/output partition of $c$. In a very real sense, therefore, a controller is regular if and only if it can be viewed as an 'intelligent controller' that processes sensor inputs outputs into actuator inputs ([10] for details).

The question arises when a controlled behavior can be implemented by a regular controller. We shall call such a controlled behavior regularly implementable. It turns out that regular implementability involves controllability [4, Chapter 5]. In fact, if $\mathcal{P}$ is controllable then every implementable $\mathcal{K}$ (i.e. $\mathcal{N} \subseteq \mathcal{K} \subseteq \mathcal{P}$ ) is regularly implementable [10], [2]. This result has recently been generalized to uncontrollable systems in [1]. Given a behavior $\mathcal{P} \in \mathfrak{L}^{*}$, we define $\mathcal{P}_{\text {controllable, the }}$ controllable part of $\mathcal{P}$ as the largest controllable sub-behavior contained in $\mathcal{P}$. The main results on regular implementability obtained in these references are summarized in the following theorem.

Theorem 3: Let $\mathcal{P}_{\text {full }} \in \mathfrak{L}^{\mathbf{v}+c}, \mathcal{P}, \mathcal{N} \in \mathfrak{L}^{\mathfrak{w}}$ be the corresponding manifest plant behavior and hidden behavior respectively, and $\mathcal{P}_{\text {controllable }}$ be the controllable part of $\mathcal{P}$. $\mathcal{K} \in \mathfrak{L}^{w}$ is regularly implementable if and only if the following conditions are satisfied:

1) $\mathcal{N} \subseteq \mathcal{K} \subseteq \mathcal{P}$

2) $\mathcal{K}+\mathcal{P}_{\text {controllable }}=\mathcal{P}$.

In particular, if $\mathcal{P}$ is controllable, then every implementable $\mathcal{K}$ is regularly implementable. Further, $\mathcal{N}$ is regularly implementable if and only if every implementable $\mathcal{K} \in \mathfrak{L}^{\mathbf{w}}$ is regularly implementable.

Note that by definition, if $\mathcal{K} \in \mathfrak{L}^{*}$ is regularly implementable, then there exists a regular controller-that implements $\mathcal{K}$. This, however, does not mean that every controller that implements $\mathcal{K}$ is a regular controller. We shall now establish below a condition under which every controller is regular. As we shall see, this is an issue that depends solely on the plant, and not on the desired controlled behavior. In fact, unless every controller is regular, every implementable controlled behavior can be irregularly implemented (for example by the canonical controller). The condition is on the control variable plant behavior $\mathcal{P}_{c} \in \mathfrak{L}^{c}$ defined as follows.

$$
\mathcal{P}_{c}:=\left\{c \mid \exists w \text { such that }(w, c) \in \mathcal{P}_{\text {full }}\right\} \text {. }
$$

In other words, $\mathcal{P}_{c}$ is obtained from $\mathcal{P}_{\text {full }}$ by eliminating $w$, and viewing the control variables $c$ as the manifest variables.

Theorem 4: Let $\mathcal{P}_{\text {full }} \in \mathfrak{L}^{\forall+c}$ be given, $\mathcal{N} \in \mathfrak{L}^{\mathbb{y}}$ and $\mathcal{P} \in \mathfrak{L}^{\mathrm{v}}$ be the hidden and the manifest plant behaviors respectively, and $\mathcal{P}_{c} \in \mathfrak{L}^{c}$ be the control variable plant behavior. Then every controller $\mathcal{C} \in \mathfrak{L}^{c}$ is regular if and only if $\mathcal{P}_{c}=\mathfrak{C}^{\infty}\left(\mathbb{R}, \mathbb{R}^{c}\right)$.

Proof: Let $R\left(\frac{d}{d t}\right) w+M\left(\frac{d}{d t}\right) c=0$ be a minimal kernel representation of $\mathcal{P}_{\text {full }}$. Note that $\mathcal{P}_{c}=\mathfrak{C}^{\infty}\left(\mathbb{R}, \mathbb{R}^{\mathrm{c}}\right)$ is equivalent to $R$ having full row rank. Suppose $\mathcal{C} \in \mathfrak{L}^{c}$ is given by a minimal kernel representation $C\left(\frac{d}{d t}\right) c=0$. Combining minimal kernel representations for $\mathcal{P}_{\text {full }}$ and $\mathcal{C}$ leads to

$$
\left[\begin{array}{cc}
R\left(\frac{d}{d t}\right) & M\left(\frac{d}{d t}\right) \\
0 & C\left(\frac{d}{d t}\right)
\end{array}\right]\left[\begin{array}{l}
w \\
c
\end{array}\right]=0
$$

a kernel representation of $\mathcal{K}_{\text {full. }}$.

(if): Suppose $\mathcal{P}_{c}=\mathfrak{C}^{\infty}\left(\mathbb{R}, \mathbb{R}^{c}\right)$, equivalently, that $R$ has full row rank. It follows that $\operatorname{rank}\left(\left[\begin{array}{cc}R & M \\ 0\end{array}\right]\right)=\operatorname{rank}(R)+$ $\operatorname{rank}(C)=\operatorname{rank}\left(\left[\begin{array}{ll}R & M\end{array}\right]\right)+\operatorname{rank}\left(\left[\begin{array}{ll}0 & C\end{array}\right]\right)$. Hence, the controller is regular.

(only if): We need to show that if every controller is regular then $\mathcal{P}_{c}=\mathfrak{C}^{\infty}\left(\mathbb{R}, \mathbb{R}^{c}\right)$. Assume, to the contrary, that $\mathcal{P}_{c} \neq$ $\mathfrak{C}^{\infty}\left(\mathbb{R}, \mathbb{R}^{c}\right)$. This implies that $R$ does not have full row rank. Then there exists an equivalent minimal kemel representation of $\mathcal{P}_{\text {full }}$ of the form

$$
\left[\begin{array}{cc}
R_{1}\left(\frac{d}{d t}\right) & M_{1}\left(\frac{d}{d t}\right) \\
0 & M_{2}\left(\frac{d}{d t}\right)
\end{array}\right]\left[\begin{array}{l}
w \\
c
\end{array}\right]=0
$$

with $R_{1}$ and $0 \neq M_{2}$ having full row rank. We see that the controller $\mathcal{C} \in \mathfrak{L}^{c}$ with minimal kernel representation $M_{2}\left(\frac{d}{d t}\right) c=0$ is a controller that is obviously not regular. This contradiction establishes that $\mathcal{P}_{\mathrm{c}}=\mathfrak{C}^{\infty}\left(\mathbb{R}, \mathbb{R}^{\mathrm{c}}\right)$.

\section{REgUlaRITY OF THE CANONICAL CONTROLLER}

We now come to the issue of regularity of the canonical controller. The following theorem shows that $\mathcal{P}_{c}=$ $\mathfrak{C}^{\infty}\left(\mathbb{R}, \mathbb{R}^{\mathrm{c}}\right)$ is a necessary and sufficient condition for $\mathcal{C}_{\text {canonical }}^{\prime}$ to be a regular controller. In other words, the canonical controller is maximally irregular: it is regular if and only if every controller is regular, and this does not depend on the desired controlled behavior that is being implemented by the canonical controller.

Theorem 5: Consider the plant $\mathcal{P}_{\text {full }} \in \mathfrak{L}^{\mathbf{u}+c}$; a desired

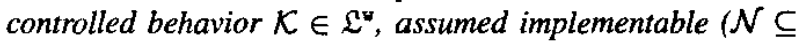
$\mathcal{K} \subseteq \mathcal{P})$, and the associated canonical controller $\mathcal{C}_{\text {canonical }}^{\prime} \in$ $\mathfrak{L}^{\mathrm{c}}$. The canonical controller implements $\mathcal{X}$ regularly if and only if $\mathcal{P}_{c}=\mathfrak{C}^{\infty}\left(\mathbb{R}, \mathbb{R}^{c}\right)$.

Proof : (if): If $\mathcal{P}_{c}=\mathfrak{C}^{\infty}\left(\mathbb{R}, \mathbb{R}^{c}\right)$, then, by the previous theorem, every controller is regular. 
(only if): Without loss of generality, assume that $\mathcal{P}_{\text {full }}$ has a minimal kemel representation of the form

$$
\mathcal{P}_{\text {full }}: \quad R_{1}\left(\frac{d}{d t}\right) w+M_{1}\left(\frac{d}{d t}\right) c=0, \quad M_{2}\left(\frac{d}{d t}\right) c=0,
$$

with $R_{1}$ and $M_{2}$ of full row rank. Since $\mathcal{N} \subseteq \mathcal{K}, \mathcal{K}$ has a minimal kernel representation of the form

$$
F\left(\frac{d}{d t}\right) R_{1}\left(\frac{d}{d t}\right) w=0 .
$$

Then, the following is a latent variable representation of the canonical behavior (with latent variable $v$ ).

$$
\begin{gathered}
\mathcal{C}_{\text {canonical }}^{\prime}: R_{1}\left(\frac{d}{d t}\right) v+M_{1}\left(\frac{d}{d t}\right) c=0, M_{2}\left(\frac{d}{d t}\right) c=0, \\
F\left(\frac{d}{d t}\right) R_{1}\left(\frac{d}{d t}\right) v=0 .
\end{gathered}
$$

Eliminating $v$ from the equations of $\mathcal{C}_{\text {canonical }}^{\prime}$ (and using the full row rank condition on $R_{1}$ ) yields a kernel representation of the canonical controller $\mathcal{C}_{\text {can }}$ of the form:

$$
M_{2}\left(\frac{d}{d t}\right) c=0 \quad F\left(\frac{d}{d t}\right) M_{1}\left(\frac{d}{d t}\right) c=0 .
$$

We see that $\mathcal{C}_{\text {can }}$ always repeats some laws of $\mathcal{P}_{\text {full, }}$, namely the rows in $M_{2}$. Thus $\mathcal{C}_{\text {can }}$ is a regular controller only if the equation $M_{2}\left(\frac{d}{d t}\right) c=0$ is absent from the equations of $\mathcal{P}_{\text {full }}$. This is equivalent to $\mathcal{P}_{c}=\mathfrak{C}^{\infty}\left(\mathbb{R}, \mathbb{R}^{c}\right)$.

Recapitulating, we have shown that the following are equivalent for a plant behavior $\mathcal{P}_{\text {full }} \in \mathfrak{L}^{\mathbf{u}+c}$ :

1) $\mathcal{P}_{c}=\mathfrak{C}^{\infty}\left(\mathbb{R} ; \mathbb{R}^{c}\right)$ : the plant control variables are free;

2) Every controller is regular;

3) The canonical controller is regular.

The condition $\mathcal{P}_{c}=\mathfrak{C}^{\infty}\left(\mathbb{R}, \mathbb{R}^{c}\right)$ is not particularly restrictive. It is satisfied in the standard LQG-like setting, with additive 'noise' surjectively entering the observed output.

\section{CONCLUSIONS}

The canonical controller is a very attractive idea, the controller par excellence that carries out internal model based thinking. We showed that it always implements an implementable controlled behavior, but that it is, unfortunately, maximally irregular. It is regular only if every controller is. One issue that is worth investigating in the future is the excessively large dynamic order of the canonical controller.

\section{ACKNOWLEDGMENTS}

This research is supported by the Belgian Federal Government under the DWTC program Interuniversity Attraction Poles, Phase V, 2002 - 2006, Dynamical Systems and Control: Computation, Identification and Modelling, by the KUL Concerted Research Action (GOA) MEFISTO-666, and by several grants en projects from IWT-Flanders and the Flemish Fund for Scientific Research.

\section{REFERENCES}

[1] M.N. Belur and H.L. Trentelman, On stabilization, pole placement and regular implementability, IEEE Transactions on Automatic Control, volume 47, pages 735-744, 2002.

[2] J.W. Polderman, Sequential continuous time adaptive control: a behavioral approach, Proceedings of 39-th IEEE Conference on Decision and Control, Sydney, Australia, pages 2484-2487, 2000.

[3] J.W. Polderman and I. Mareels, A behavioral approach to adaptive control, in: Mathematics of System and Control , J.W. Polderman and H.L. Trentelman eds., Festschrift on the occasion of the 60-th birthday of J.C. Willems, ISBN 90-367-1112-6, Groningen, pages 119-130, 1999.

[4] J.W. Polderman and J.C. Willems, Introduction to Mathematical Systems Theory, a Behavioral Approach, Springer Verlag, 1997.

[5] A.J. van der Schaft and A.A. Julius, Achievable behavior by composition, Proceedings of 41-th IEEE Conference on Decision and Control, Las Vegas, Nevada, pages 7-12, 2002.

[6] A.J. van der Schaft, Achievable behavior of general systems, Systems \& Control Letters, volume 49, pages 141-149, 2003.

[7] H.L. Trentelman, A truly behavioral approach to the $\mathcal{H}_{\infty}$ control problem, in: Mathematics of System and Control, J.W. Polderman and H.L. Trentelman eds., Festschrift on the occasion of the 60-th birthday of J.C. Willems, ISBN 90-367-1112-6, Groningen, pages $177-190,1999$.

[8] H.L. Trentelman and J.C. Willems, Synthesis of dissipative systems using quadratic differential forms: Part II, IEEE Transactions on Automatic Control, volume 47, pages 70-86, 2002.

[9] J.C. Willems, Paradigms and puzzles in the theory of dynamical systems, IEEE Transactions on Automatic Control, volume 36, pages 259-294, 1991.

[10] J.C. Willems, On interconnections, control, and feedback, IEEE Transactions on Automatic Control, volume 42, pages 326-339, 1997.

[11] J.C. Willems, Behaviors, latent variables, and interconnections, Systems, Control and Information (Japan), volume 43, pages 453-464, 1999.

[12] J.C. Willems and H.L. Trentelman, Synthesis of dissipative systems using quadratic differential forms: part I, IEEE Transactions on Automatic Control, volume 47, pages 53-69, 2002.

[13] www.esat. kuleuven.ac.be/ jwillems 